\title{
Prevention of vertical HIV transmission and management of the HIV-exposed infant in Canada in 2014
}

\author{
Ari Bitnun MD MSC FRCPC 1 , Jason Brophy MD MSc FRCPC ${ }^{2}$, Lindy Samson MD MSc FRCPC ${ }^{2}$, Ariane Alimenti MD ${ }^{3}$, \\ Fatima Kakkar MD MPH FRCPC ${ }^{4}$, Valerie Lamarre MD FRCPC ${ }^{4}$, Dorothy Moore MD PhD, FRCPC \\ Christos Karatzios MD FRCPC ${ }^{4,5}$, Sandra Seigel MD FRCPC ${ }^{6}$, Laura Sauve MD MPH DTM\&H ${ }^{3}$, Wendy Vaudry MD CM FRCPC ${ }^{7}$ \\ Mark H Yudin MD MSc FRCSC ${ }^{8}$, Deborah Money MD FRCSC ${ }^{9}$; for the Canadian Paediatric and Perinatal AIDS Research \\ Group and the Infectious Diseases Committee of the Society of Obstetricians and Gynaecologists of Canada
}

\begin{abstract}
$\mathrm{T}$ he standard of care for the prevention of vertical transmission (VT) of HIV in Canada and other developed countries includes routine prenatal HIV testing for all pregnant women, and for those testing positive: antepartum combination antiretroviral therapy (cART); intrapartum intravenous zidovudine; six weeks of postnatal oral zidovudine to the infant; and exclusive formula feeding of the infant. With these interventions, the rate of VT has been reduced from $25 \%$ to $40 \%$, to $<2 \%$ in many jurisdictions (1-3). Data from the Canadian Perinatal HIV Surveillance Program indicate that, in Canada, the VT rate declined from $19.6 \%$ between 1990 and 1996 to 2.5\% between 1997 and 2012. During the latter period, the VT rate was $0.1 \%$ when mothers were prescribed cART $>4$ weeks before delivery. The purpose of the present article is to highlight recent changes in management guidelines and important caveats to these changes with regard to prevention of VT in the Canadian context. There is a global trend to replace the use of the previous conventional terminology of 'mother-to-child transmission' with 'vertical transmission' or 'perinatal transmission' to remove implications of blame from the mother. In the present article, 'vertical transmission' is used throughout.
\end{abstract}

\section{HIV testing during pregnancy}

HIV testing is recommended for all pregnant women in Canada, with appropriate pre- and post-test counselling. Women whose HIV status is unknown at the time of delivery should undergo rapid HIV antibody testing. For women at increased risk for HIV infection (eg, intravenous drug use, commercial sex work, frequent unprotected intercourse with multiple partners, HIV-negative woman of a serodiscordant couple) who test negative early in pregnancy, repeat testing late in pregnancy (beginning of the third trimester) and at delivery is strongly encouraged. When such women present in labour, advice regarding maternal HIV diagnosis and management should be sought from obstetric and adult HIV experts on an urgent basis; similarly, pediatric HIV experts should be consulted regarding infant management in such situations.

Women living with HIV, either previously diagnosed or identified during pregnancy, should be followed by a specialist with expertise in the management of HIV during pregnancy, treated with cART and monitored for viral suppression. Prelabour elective Cesarean section delivery should be planned for those not on cART and/or anticipated or documented to have inadequate viral suppression near delivery (viral load $>1000$ copies $/ \mathrm{mL}$ ).

For women who were previously diagnosed with HIV in whom viral load is not documented to be fully suppressed in the four weeks preceding onset of labour and for women diagnosed with HIV infection at the time of labour, urgent consultation with adult and pediatric HIV experts and an obstetrician with expertise in the management of HIV is essential.

\begin{abstract}
Recommendation 1: Pediatricians and other health care providers involved in the care of HIV-infected pregnant women and their children should advocate for universal HIV testing of all pregnant women with appropriate pre- and post-test counselling so that appropriate preventive measures can be implemented in a timely manner. When this fails, testing of the mother at delivery, or of the infant if maternal testing is not possible, should be ensured. Ideally, no infant should be discharged from hospital without the HIV status of the mother being known. Pediatricians and family physicians who perform routine neonatal examinations should verify that the HIV status of the mother or child is known and documented.

Recommendation 2: Urgent consultation with an obstetrician with HIV expertise and an adult HIV expert is recommended for diagnosis and management of women deemed to be at high risk for HIV infection with unknown status at the time of labour. Similarly, a pediatric HIV expert should be consulted regarding infant management in such situations.
\end{abstract}

\section{Intravenous zidovudine during labour}

The United States Department of Health and Human Services (DHHS) guidelines now indicate that intrapartum intravenous zidovudine is not required for HIV-infected women who received cART during pregnancy and had an HIV viral load $<400$ copies $/ \mathrm{mL}$ near the time of delivery (4). This recommendation was based on data from the French Perinatal Cohort demonstrating transmission rates of $0.6 \%$ (17 of 2750 ) and $0 \%$ (zero of 95 ) with and without intrapartum intravenous zidovudine, respectively, among women who received antenatal cART and had a viral load $<400$ copies/mL before delivery (3). However, this strategy has not been evaluated in a randomized controlled trial. Furthermore, for many women, knowledge of viral suppression and consistency of antiretroviral medication adherence cannot be confirmed. British Columbia data indicate that approximately $9 \%$ of women with documented undetectable viral load antenatally will be found retrospectively to have detectable viral load at delivery (5).

Recommendation: The Canadian Paediatric and Perinatal AIDS Research Group (CPARG) and the Infectious Diseases Committee of the Society of Obstetricians and Gynaecologists of Canada (ID-SOGC) continue to recommend intravenous zidovudine for all HIV-infected women in labour regardless of antepartum ART regimen, mode of delivery or viral load near delivery.

${ }^{1}$ Department of Paediatrics, Hospital for Sick Children, University of Toronto, Toronto; ${ }^{2}$ Department of Paediatrics, Children's Hospital of Eastern

Ontario, University of Ottawa, Ottawa, Ontario; ${ }^{3}$ Department of Paediatrics, British Columbia Women's Hospital and Health Centre,

University of British Columbia, Vancouver, British Columbia; ${ }^{4}$ Department of Paediatrics, Centre hospitalier universitaire Sainte-Justine,

Université de Montréal; ${ }^{5}$ Department of Paediatrics, Montreal Children's Hospital, McGill University, Montreal, Quebec; ${ }^{6}$ Department of Paediatrics, Hamilton Health Sciences, McMaster University, Hamilton, Ontario; ${ }^{7}$ Department of Paediatrics, Stollery Children's Hospital, University of Alberta, Edmonton, Alberta; ${ }^{8}$ Department of Obstetrics and Gynecology, St Michael's Hospital, University of Toronto, Toronto, Ontario; ${ }^{9}$ Department of Obstetrics and Gynecology, Women's Health Research Institute, British Columbia Women's Hospital and Health Centre, University of British Columbia, Vancouver, British Columbia

Correspondence and reprints: Dr Ari Bitnun, University of Toronto, Division of Infectious Diseases, Department of Paediatrics, The Hospital for Sick Children, 555 University Avenue, Toronto, Ontario M5G 1X8. Telephone 416-813-7654 ext 204649, fax 416-813-8404, e-mail ari.bitnun@sickkids.ca 
TABLE 1

Recommendations for antiretroviral prophylaxis in infants of HIV-infected mothers

\begin{tabular}{|c|c|}
\hline Antenatal maternal treatment & Recommended antiretroviral treatment \\
\hline $\begin{array}{l}\text { Administered; documented viral } \\
\text { load }<40 \text { copies } / \mathrm{mL}\end{array}$ & Zidovudine for six weeks \\
\hline $\begin{array}{l}\text { Administered, but viral load } 40 \text { copies } / \mathrm{mL} \text { to } \\
999 \text { copies/mL }\end{array}$ & $\begin{array}{l}\text { Zidovudine for six weeks OR } \\
\text { Zidovudine for six weeks PLUS three doses of nevirapine during first week of life OR combination antiretroviral therapy }\end{array}$ \\
\hline Administered, but viral load $\geq 1000$ copies $/ \mathrm{mL}$ & Zidovudine for six weeks PLUS three doses of nevirapine during first week of life OR combination antiretroviral therapy \\
\hline Not administered & Zidovudine for six weeks PLUS three doses of nevirapine during first week of life OR combination antiretroviral therapy \\
\hline
\end{tabular}
triple combination antiretroviral therapy for neonates born to such mothers

\section{TABLE 2}

Currently approved zidovudine and nevirapine dosing for neonates for the prevention of vertical HIV transmission

\begin{tabular}{|c|c|c|}
\hline Medication & $\begin{array}{l}\text { Gestational age, } \\
\text { weight at birth }\end{array}$ & Dosing \\
\hline \multirow[t]{6}{*}{ Zidovudine } & $\geq 35$ weeks & Oral: 4 mg/kg/dose twice daily for six weeks OR \\
\hline & & Intravenous: $3 \mathrm{mg} / \mathrm{kg} /$ dose every $12 \mathrm{~h}$ (if unable to tolerate oral medication) for six weeks \\
\hline & 30 weeks to & Oral: $2 \mathrm{mg} / \mathrm{kg} / \mathrm{dose}$ twice daily for two weeks, followed by $3 \mathrm{mg} / \mathrm{kg} / \mathrm{dose}$ twice daily for four weeks OR \\
\hline & $<35$ weeks & $\begin{array}{l}\text { Intravenous: } 1.5 \mathrm{mg} / \mathrm{kg} / \text { dose every } 12 \mathrm{~h} \text { for first two weeks of life and } 2.3 \mathrm{mg} / \mathrm{kg} / \mathrm{dose} \text { every } 12 \mathrm{~h} \text { from } 15 \text { days to six weeks of life } \\
\text { (if unable to tolerate oral medication) }\end{array}$ \\
\hline & $\leq 30$ weeks & Oral: $2 \mathrm{mg} / \mathrm{kg} / \mathrm{dose}$ twice daily for four weeks, followed by $3 \mathrm{mg} / \mathrm{kg} / \mathrm{dose}$ twice daily for two weeks OR \\
\hline & & $\begin{array}{l}\text { Intravenous: } 1.5 \mathrm{mg} / \mathrm{kg} / \text { dose every } 12 \mathrm{~h} \text { for first four weeks of life and } 2.3 \mathrm{mg} / \mathrm{kg} / \mathrm{dose} \text { every } 12 \mathrm{~h} \text { from four to six weeks of life } \\
\text { (if unable to tolerate oral medication) }\end{array}$ \\
\hline \multirow[t]{2}{*}{ Nevirapine } & $>2 \mathrm{~kg}$ & $\begin{array}{l}\text { Oral: Three doses (12 mg/dose): first dose within first } 48 \mathrm{~h} \text { of life (birth to } 48 \mathrm{~h} \text { ), second dose } 48 \mathrm{~h} \text { after the first dose and third } \\
\text { dose } 96 \mathrm{~h} \text { after the second dose }\end{array}$ \\
\hline & $\begin{array}{l}1.5 \mathrm{~kg} \text { to } \\
2.0 \mathrm{~kg}\end{array}$ & $\begin{array}{l}\text { Oral: Three doses ( } 8 \mathrm{mg} / \text { dose): first dose within first } 48 \mathrm{~h} \text { of life (birth to } 48 \mathrm{~h} \text { ), second dose } 48 \mathrm{~h} \text { after the first dose and third dose } \\
96 \mathrm{~h} \text { after the second dose }\end{array}$ \\
\hline
\end{tabular}

Adapted from guidelines for the use of antiretroviral agents in pediatric HIV infection, Department of Health and Human Services, United States (4). Urgent consultation with a pediatric HIV expert is recommended for all HIV-exposed infants in whom dual or triple antiretroviral therapy is being considered; the choice of antiretroviral agents and dosing depends on multiple factors; regimens used vary from site to site

Antiretroviral medications for the infant (Tables 1 and 2)

All infants of mothers with HIV infection should receive antiretroviral therapy beginning as soon as possible after birth, preferably within $6 \mathrm{~h}$ to $12 \mathrm{~h}$ of birth. Efficacy decreases with increasing time after birth and is likely completely lost when initiated beyond $72 \mathrm{~h}$ of life.

For infants whose mothers received cART during pregnancy and had an undetectable viral load before delivery, zidovudine monotherapy from birth to six weeks of life remains the standard of care. Based on pharmacokinetic data, the dosing of oral zidovudine recommended in the DHHS guidelines was recently revised (4).

Recommendation 1: Consultation with a pediatric HIV expert is strongly recommended for all newborn infants of HIV-infected mothers.

Recommendation 2: CPARG recommends antiretroviral therapy for all HIV-exposed newborns and supports the DHHS dosing guidelines for oral zidovudine (Tables 1 and 2) (4).

The management of infants born to mothers with suboptimal control of HIV replication at the time of delivery is complex. The DHHS guidelines support the combination of zidovudine for six weeks and three doses of nevirapine during the first week of life for infants of mothers who did not receive antenatal antiretroviral drugs (4). However, cART, most often with zidovudine, lamivudine and nevirapine, is advocated by many experts in the field. In Canada, treatment strategies for such 'highrisk situations' vary and are based on expert opinion.

Recommendation 3: Urgent consultation with a pediatric HIV expert is essential when managing a newborn infant of an HIV-infected mother in the following circumstances: the mother did not consistently receive cART during pregnancy regardless of the reason (lack of antenatal care, poor adherence, etc); the mother's most recent viral load was either detectable ( $\geq 40$ copies $/ \mathrm{mL}$ ) or was not documented in the four weeks preceding delivery; or the mother did not receive intrapartum prophylaxis. Consultation before delivery, if possible, is ideal to gather information and formulate a treatment plan.
Recommendation 4: Postexposure prophylaxis of infants with triple cART or zidovudine plus nevirapine is recommended in situations in which the mother's viral load is known to be elevated or suspected to be elevated on the basis of no antenatal therapy, poor adherence to such therapy or other information. Such treatment should be started as soon as possible after delivery and no later than $72 \mathrm{~h}$ of life. The specific combination of antiretroviral medications to be used and medication dosing should be determined in consultation with a local pediatric HIV expert.

The DHHS guidelines indicate that lopinavir/ritonavir (Kaletra, Abbott Laboratories, USA) should not be used in infants in the immediate postnatal period due to the potential for life-threatening toxicity (4). Reported toxicities in premature infants, from the drug itself and/or inactive ingredients in the oral solution (propylene glycol, ethanol), include: transient symptomatic adrenal insufficiency; lifethreatening bradyarrhythmias and cardiac dysfunction; and lactic acidosis, acute renal failure, central nervous system depression and respiratory depression.

Recommendation 5: Lopinavir/ritonavir (Kaletra) should not be used in infants until a postmenstrual age of 42 weeks and a postnatal age of at least 14 days.

\section{Infant feeding}

Several randomized clinical trials in Africa have shown that breastmilk transmission of HIV was curtailed substantially when nursing mothers received ongoing cART postnatally $(6,7)$. However, HIV transmission did occur in a small number of cases despite undetectable viral load in maternal serum and breast milk. Furthermore, the reduction in mortality from diarrheal and respiratory tract diseases attributable to breastfeeding in sub-Saharan Africa is not applicable to the Canadian context.

Social pressure to breastfeed is common in many communities and failure to do so can be perceived by some to be indicative of HIV infection 
in the mother. Therefore, women need to be supported and provided with advice as to how to explain the choice to bottle feed without disclosing their HIV status. Counselling on this issue is essential and best initiated before delivery by a health care professional or community support worker experienced in working with HIV-infected pregnant women and mothers. Ongoing support is often required. If an HIV-infected woman is found to be breastfeeding, she and her child should be referred urgently to a pediatric HIV expert. It is important to clarify the mother's understanding of the risks of HIV transmission via breast milk and to determine the reasons for her choosing to breastfeed. An automatic referral to child protection services is not warranted (8), but may be considered in some instances after consultation with a pediatric HIV specialist.

Premastication of food by HIV-infected caregivers has recently been implicated as a potential route of HIV transmission to young infants (9).

Recommendation 1: CPARG and ID-SOGC continue to recommend exclusive formula feeding of infants born to HIV-infected mothers.

Recommendation 2: CPARG and ID-SOGC strongly recommend that all provinces and territories provide free formula to all infants of HIV-infected mothers for the first 12 months of life.

Recommendation 3: CPARG and ID-SOGC recommend consultation with a pediatric HIV expert if an HIV-infected mother is found to be breastfeeding her infant. Personal and/or cultural beliefs surrounding breastfeeding should be explored, and any barriers to formula feeding identified to best support mothers in pursuing formula feeds.

Recommendation 4: CPARG and ID-SOGC recommend inquiring about premastication of food by caregivers with HIV infection and advising against this practice because of the potential for HIV transmission.

\section{Follow-up of HIV-exposed infants and children}

HIV-exposed infants need to be followed closely both to determine their HIV status and to monitor for potential toxicity of antiretroviral medications.

HIV DNA and/or RNA polymerase chain reaction (PCR) are the preferred assays used for diagnosis of HIV infection in infants $<18$ months of age. Some Canadian laboratories are using a qualitative PCR assay that tests for viral RNA as well as proviral DNA. However, most provinces are using quantitative RNA PCR (viral load) testing; for infants receiving cART, reliance on such tests to exclude HIV infection early in infancy is potentially problematic. A good understanding of the limitations of the assays being used is, therefore, important in clinical care. Involvement of a pediatric HIV expert is warranted for positive or indeterminate results.

Transplacental maternal antibody will clear in $25 \%$ of HIVexposed infants by 12 months of age, but should clear in all such

\section{REFERENCES}

1. Forbes JC, Alimenti AM, Singer J, et al. A national review of vertical HIV transmission. AIDS 2012;26:757-63.

2. Townsend CL, Cortina-Borja M, Peckham CS, de Ruiter A, Lyall H, Tookey PA. Low rates of mother-to-child transmission of HIV following effective pregnancy interventions in the United Kingdom and Ireland, 2000-2006. AIDS 2008;22:973-81.

3. Warszawski J, Tubiana R, Le Chenadec J, et al. Mother-to-child HIV transmission despite antiretroviral therapy in the ANRS French Perinatal Cohort. AIDS 2008;22:289-99.

4. Panel on Treatment of HIV-Infected Pregnant Women and Prevention of Perinatal Transmission. Recommendations for Use of Antiretroviral Drugs in Pregnant HIV-1-Infected Women for Maternal Health and Interventions to Reduce Perinatal HIV Transmission in the United States. <http://aidsinfo.nih.gov/ contentfiles/lvguidelines/PerinatalGL.pdf $>$

(Accessed February 3, 2014). infants by 18 to 24 months of life. Newer-generation HIV antibody tests are more sensitive and may continue to detect low levels of maternal antibody beyond 18 months of age. Documentation of seroreversion is important as an international standard for lack of vertical transmission and to detect possible late transmission from breastfeeding. Every effort should be made to counsel parents about the significance of these tests to minimize undue anxiety while awaiting results of tests for seroreversion.

Recommendation 1: Qualitative or quantitative HIV DNA or RNA PCR is recommended for diagnosis of HIV infection in children $<18$ months of age. Due to assay limitations, CPARG recommends involvement of a pediatric HIV expert to aid in the interpretation of test results.

Recommendation 2: For most infants, HIV infection can be reasonably excluded with two separately timed negative PCR tests, one taken at or beyond one month of age and the second at or beyond two months of age. For children who received cART, at least one of the PCR tests should be performed at or beyond four months of age. Recommendation 3: The HIV status of exposed infants should be finalized using a serological assay between 18 and 24 months of age.

Infants exposed in utero and perinatally to antiretroviral medications need to be monitored for potential short- as well as long-term toxicity regardless of HIV status. Reversible anemia and neutropenia are the most common acute side effects observed with zidovudine; occasionally this may necessitate early discontinuation of the drug. Long-term toxicity of the nucleoside analogues mediated by inhibition of mitochondrial DNA polymerase gamma and telomerase is of potential, although unproven, concern. Infants who develop significant organ dysfunction of unknown etiology while on or after recent exposure to nucleoside analogues should be evaluated for potential mitochondrial dysfunction. Long-term follow-up and annual physical examinations, into adulthood, of HIV-uninfected infants exposed in utero and perinatally to antiretroviral medications is now recommended by the DHHS because of the potential carcinogenicity of the nucleoside analogues (4). Finally, it is important to ensure continued psychosocial support for HIV-exposed uninfected children and their families.

Recommendation 4: CPARG supports the DHHS recommendation for long-term follow-up of all children exposed in utero and perinatally to antiretroviral medications. Such follow-up can be undertaken by any physician familiar with the potential long-term risks of in utero and perinatal antiretroviral exposure.

ACKNOWLEDGEMENTS: This article has been endorsed by the Canadian Paediatric Society.

5. Money DM, van Schalkwyk J, Alimenti A, et al. HIV viral rebound near delivery in previously suppressed, combination antiretroviral therapy (cART) treated pregnant women (Abstract O037). Can J Infect Dis Med Microbiol 2013;24:20A.

6. Chasela CS, Hudgens MG, Jamieson DJ, et al. Maternal or infant antiretroviral drugs to reduce HIV-1 transmission. N Engl J Med 2010;362:2271-81.

7. Shapiro RL, Hughes MD, Ogwu A, et al. Antiretroviral regimens in pregnancy and breast-feeding in Botswana. N Engl J Med 2010;362:2282-94.

8. Taylor GP, Clayden P, Dhar J, et al. British HIV Association guidelines for the management of HIV infection in pregnant women 2012. HIV Med 2012;13 Suppl 2:87-157.

9. Gaur AH, Dominguez KL, Kalish ML, et al. Practice of feeding premasticated food to infants: A potential risk factor for HIV transmission. Pediatrics 2009;124:658-66. 


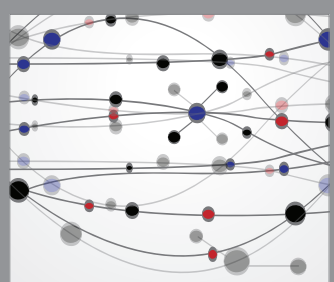

The Scientific World Journal
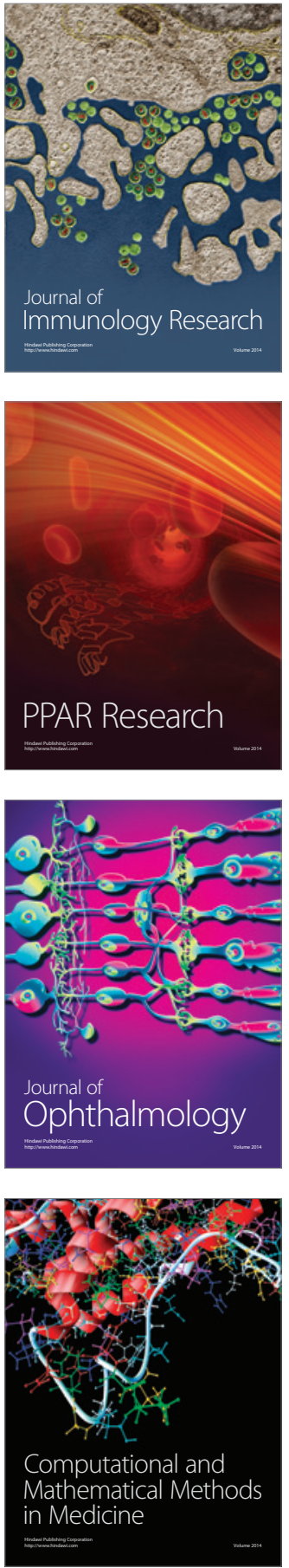

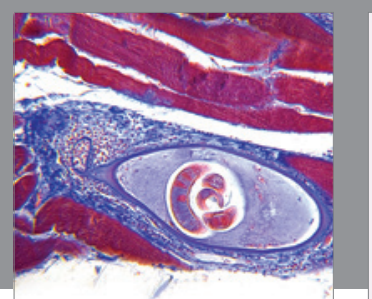

Gastroenterology Research and Practice

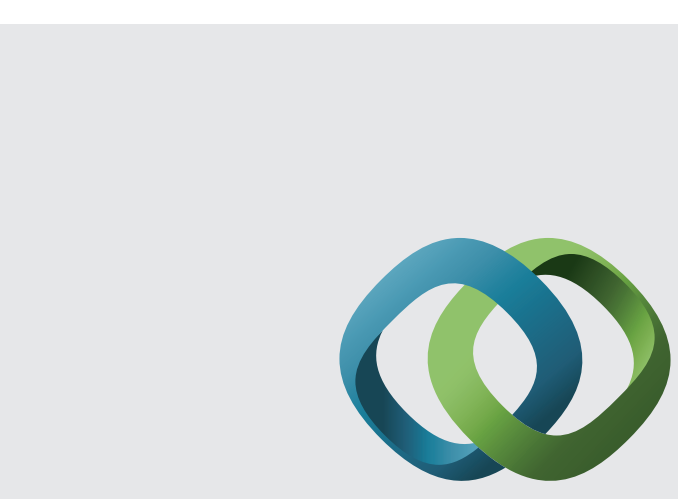

\section{Hindawi}

Submit your manuscripts at

http://www.hindawi.com
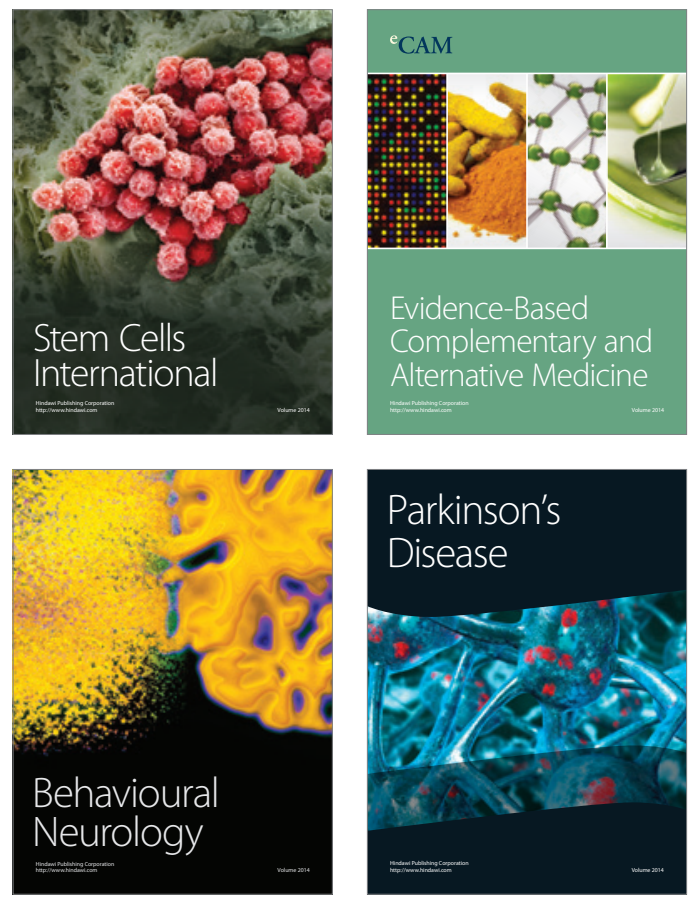
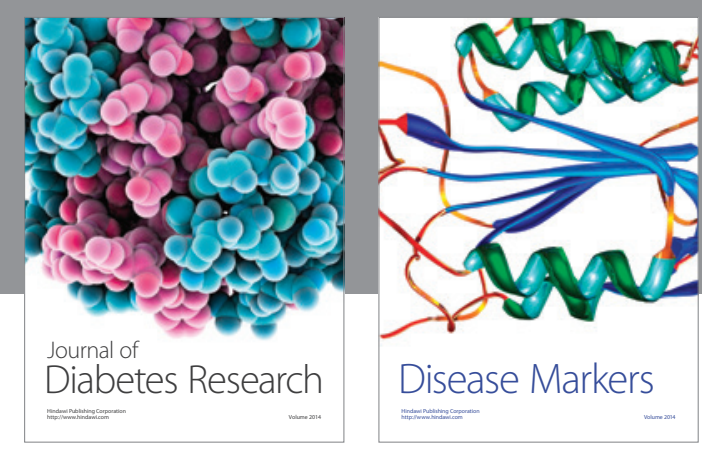

Disease Markers
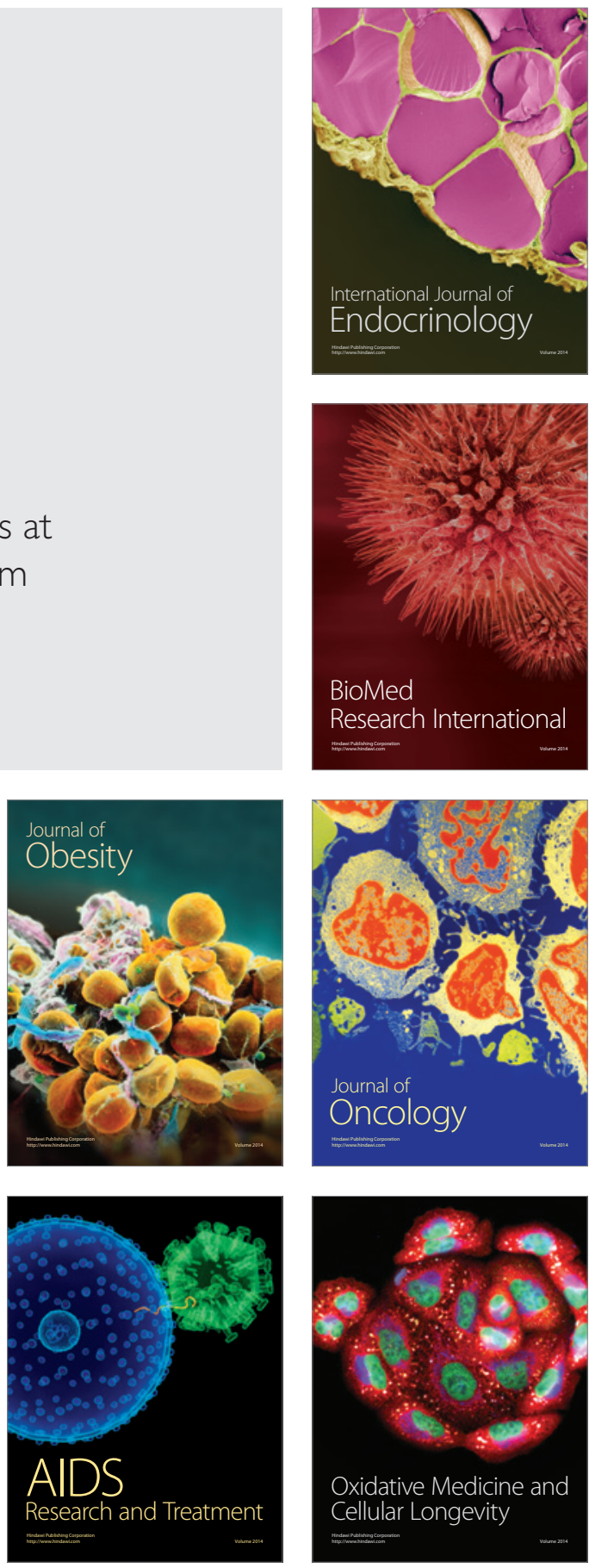